\title{
Considerations Regarding The Rehabilitation of Oncological Patients With Neuropathies
}

\author{
MICU Elena Claudia ${ }^{1}$, IRSAY Laszlo ${ }^{1,2}$
}

\author{
${ }^{1}$ Clinical Rehabilitation Hospital Cluj-Napoca \\ 2 University of Medicine and Pharmacy Cluj-Napoca
}

\begin{abstract}
The most common reason for which oncological patients come in the rehabilitation clinics, is pain. This can have various etiologies which are related or not to the neoplasia. In both cases, pharmacotherapy and the physical-kinetic-therapy should be individualized. Over the time, the therapeutic approach for these patients varied due to the fear of not harming. Nowadays, we can count on a few studies when applying various medical treatments and physical-kinetic modalities. An interdisciplinary team should collaborate to treat the pain during the course of illness of an oncological patient. The availability of professionals from major disciplines is essential to offering comprehensive care.

However, each specialist must know their medical field in order to give the patient the best treatment. Thus, doctors in other specialties than physical medicine and rehabilitation should provide a more accurate diagnosis of neoplasia as co-present diseases, so the rehabilitation doctors to prescribe proper physical-kinetic procedures and rehabilitation modalities.
\end{abstract}

Keywords: pain, neoplasia, rehabilitation

\section{Introduction}

The pain in cancer patients, although common is not easy to treat as it requires a wide range of analgesic drugs, from non opioid ones to morphine derivatives. Interventional techniques as: nerve blockade or intrathecal administration associated with systemic medication are being used in $10 \%$ of cases [1]. In cancer patients, the classification of pain is very important for the symptomatic treatment.

The pain can be due to the presence of neoplasia/treatment of neoplasia, or not. Chemotherapy is associated with neurotoxicity and peripheral polyneuropathy (PNP). The latter occurs in $70-100 \%$ of patients treated with chemotherapy agents administered in large doses and in $12 \%$ of patients treated with chemotherapy agents administered in minimum doses. [2]

Many patients with cancer, particularly those with advanced or metastatic disease, have increased levels of pain. [3]
Osteonecrosis and reduced bone mineral density are common among patients with cancer. Both cancer and cancer treatments increase the risk for developing osteonecrosis in a variety of locations, including proximal or distal femur, proximal humerus, jaw, and metatarsals. [4]

New-onset pain and decreased weight-bearing ability should alert therapists to the possibility of osteonecrosis; however, this condition is not always symptomatic. [5]

Both radiation and chemotherapy can alter the structure and function of the central nervous system and may result in impaired mental function in patients during or following treatment for their cancer. [6]

Emotional functions also may affect the ability of a patient to participate in the physical therapy intervention. A tool that has been used to evaluate emotional functions in patients with cancer is the 
Profile of Mood States. This self-report instrument is easy to use and may provide insight into our patient's ability to respond to and participate in a physical therapy program. [7]

Pharmacotherapy is associated with the use of opioid and/or non opioid and adjuvant analgesics, the latter being divided into 3 groups: antidepressants (e.g. amitriptyline, venlafaxine, duloxetine), anticonvulsants (e.g. sodium valproate, phenytoin, carbamazepine) and local anesthetics (e.g. lidocaine) [8]. The guidelines have decided gabapentin (a medicine used in antiepileptic treatments) as the first medicine to be used. [9]

Capsaicin is another method used to treat pain, one which has not been studied on oncological patients. Studies on capsaicin have been conducted within the treatment in diabetic patients. However, the results were inconclusive and therefore this course of treatment is not recommended at present. [10]

If the pain is due to nerve compression steroids should be considered. There is evidence that I.V. Lidocaine and its oral correspondent, Mexiletine, constitute a more effective method to reduce neuropathic pain than placebo in adult oncological patients and can alleviate the pain in selected patients [11]. There may be two or more pain etiologies in one oncological patient. The treatment method may come with relative or absolute contraindications regarding the oncological etiology (e.g. vitamin supplements).

\section{Are vitamins beneficial or increase the risk?}

B vitamins interfere with cell metabolism (whether healthy or affected by neoplasia). Vitamin B1 (thiamine) is a coenzyme involved in the carbohydrate metabolism and the nervous function; vitamin B2 (riboflavin) and B3 (niacin) are coenzymes integrated in the cycle of citric acid, the metabolism of fat acids and the transportation of electrons. Vitamin B12 is a coenzyme involved in the metabolism of folic acid and the nervous function. Folic acid is a coenzyme involved in the synthesis of DNA and RNA acids.

The intake of vitamin B6 lowers the risk of developing colorectal cancer [12].

A study conducted on vitamins B6 and B12 in Finland revealed a significantly lower risk of developing a pulmonary neoplasm in patients with high vitamin B6 levels. Therefore, vitamin B6 plays a protective role against pulmonary neoplasms as well [13].

\section{Treatment of oncological patients. Studies conducted on physical procedures Objectives:}

Pain management

Maintaining or improving joint mobility

Maintaining or improving muscular force

Gait training

Easing the performance of activities of daily living (ADL)

Improving life quality

Transcutaneous electrical stimulation (TENS) is the physical procedure most frequently used in the treatment of pain regardless of the etiology. No studies on TENS have been conducted on patients presenting with neoplasia. A study revealed an improvement of the numb sensation, stinging pain and allodynia associated with modifications in the perception of vibrations and temperature and a reduction of the pain threshold by means of TENS therapy. Another study revealed that high-frequency muscle stimulation is more effective than TENS as far as the improvement of pain evaluation scores and peripheral neuropathy symptom evaluation are concerned. However, both proved effective in the alleviation of symptoms. No risks regarding the 
application of TENS therapy in patients presenting with neoplasia have been reported [10].

LASER light radiation therapy is also an option, depending on the case particular features. This treatment is associated with the fewest relative or absolute contraindications. No studies that advise against the use of LASER therapy in oncological patients have been found.

Both therapies are locally contraindicated.

Hydrogymnastics in a pool at a neutral temperature of $35-37^{\circ} \mathrm{C}+/$ - herbs. This technique causes an improvement in the central and peripheral blood flow and stimulates the cardiorespiratory capacity. Demonstrated effects include the increase of the nerve sensitivity threshold and the stimulation of the metabolism [14].

No studies advising against hydrotherapy in patients presenting with neoplasia are known at present!

Thermal and electrotherapy are avoided on account of their stimulation of the blood flow and cell metabolism (healthy cells and by analogy, cells possibly affected by neoplasia). Precaution is recommended despite the absence of studies to demonstrate any possible negative effects. The absence of studies explains the fact that these therapeutic methods are associated with relative contraindications rather than with noticed harmful effects.

Occupational therapy improves fine movements and the reeducate perception. It uses vibrating platforms and balance systems as support devices. Occupational therapists evaluate patients' ability to carry out tasks related to self-care, including activities of daily living (ADLs), such as dressing, bathing, meal preparation, and homemaking. These professionals also assist patients to increase ability to perform ADLs, including the use of compensatory techniques and adaptive equipment. In addition, occupational therapists evaluate home environments for potential modification, and they provide instruction in driving with adaptive devices. They implement interventions to promote upperextremity ROM, strength, endurance, and coordination [15].

Kinetotherapy is applied in parallel with the training in compensatory strategies and the proper use of support devices and ortheses with the purpose of obtaining maximum autonomy during occupational and physical therapy. Motor deficiency is a common complication associated with neuropathy in oncological patients. They can benefit from kinetotherapy (but the intensity and duration must be correlated with the patient's condition). Self care is sought in intermediary/advanced stages.

Compensatory strategies are applied as support for orthostatism, transfers or ambulation. Stimulation and energy preservation strategies can also be useful in patients dealing with severe fatigue. The patient is taught to adjust their posture and alter their biomechanics in order to maximize their strength and preserve their muscle groups.

Stretching is used for the antagonists of the muscles affected. Ortheses can also be used to maintain posture. Also within kinetotherapy, physiotherapists must insist on muscle toning exercises with or without the association of bio-feedback techniques, proprioception facilitation or stimulation.

Gait training is conducted using walking devices if the pain or muscular deficiency does not allow orthostatism or ambulation.

The majority of development groups extrapolated their results starting from studies on non-oncological patients in 
order to formulate recommendations. Therefore, these guides do not provide solutions to important situations, such as altered kinetics and side effects in these patients.

The supply of proper support devices is critical for the therapy to be successful. There are no studies related to the utilization of support devices in patients presenting with neoplasia. However, two non-randomized trials have been conducted on the use of canes and ortheses in diabetic patients. These devices proved efficient in the prevention of falls, improvement of balance and proprioception [10].

Acupuncture is another alternative treatment method.

While there is no cure for lymphedema, the condition can be managed through a combination of early detection, clinical treatment, education, and home management. In the early stages of lymphedema, when very mild swelling is present, the condition is managed by compression garment wear, exercise, and elevation. If the condition progresses, physical therapist management may include:

- Manual lymph drainage (to help improve the flow of lymph from the affected arm or leg)

- Compression garment wear following lymphatic drainage.

- Skin care (such as cleaning the skin of the arm or leg daily and moisten with lotion).

- Exercise to improve cardiovascular health and help decrease swelling in some cases.

- Patient education (proper diet to decrease fluid retention; how to avoid injury/ infection).

- Garment fitting [16].
Patients and their families often have a number of psychological and adjustment issues related to the illness, its treatment, and its resulting disabilities. The psychologist assesses and treats patients to help them manage their cancer-related psychological distress. As a member of the rehabilitation team, the psychologist assists other team members when psychological issues, either in patients or their family members, complicate efforts to provide effective therapy. The goal of consulting the psychologist is to maximize the benefit the patient derives from rehabilitation [15].

A Danish study determined that compared with the general population, a greater percentage of individuals who have been diagnosed with cancer are hospitalized for depression. According to the report, which investigated depressionrelated hospitalizations occurring between 1973 and 2003, the relative risk for depression in the first year after an individual had been diagnosed with cancer ranged from 1.16 (in women with colorectal cancer) to 3.08 (in men who had been diagnosed with brain cancer). The authors concluded that depression must be recognized early and treated effectively in persons who have been diagnosed with cancer in order to avoid the need to hospitalize these individuals for depression [15]. 


\section{References}

[1]. Ripamonti CI, Santini D, Maranzano E, Berti M, Roila F, ESMO Guidelines Working Group. Management of cancer pain: ESMO Clinical Practice Guidelines. Oxford Journals. Medicine. Ann Oncol. 2012;23 Suppl 7: vii139-154.

[2]. Weimer LH. Medication-Induced Neuropathies. Curr Neurol. Neurosci Rep. 2003;3(1): 86-92.

[3]. Cherny N. The management of cancer pain. CA Cancer J Clin. 2000;50:70-116. Available from:

http://www.ncbi.nlm.nih.gov/pubmed/1087048 7 - accessed in 29th july 2014

[4]. Majhail N, Ness K, Burns L, et al. Late effects in survivors of Hodgkin and nonHodgkin lymphoma: a report from the Bone Marrow Transplant Survivor Study. Biol Blood Marrow Transplant. 2007;13:1153-1159. Available from: http://www.ncbi.nlm.nih.gov/pmc/articles/PM C2083636/- accessed in 29th july 2014

[5]. Marchese VG, Connolly B, Able C, et al. Relationships among severity of osteonecrosis, pain, range of motion, and functional mobility in children, adolescents, and young adults with acute lymphoblastic leukemia. Phys Ther. 2008;88:341-350. Available from: http://www.ncbi.nlm.nih.gov/pubmed/1820207 9- accessed in 29th july 2014

[6]. Ahles T, Saykin A. Breast cancer chemotherapy-related cognitive dysfunction. Clin Breast Cancer. 2002;3:S84-S90.

Available from:

http://www.ncbi.nlm.nih.gov/pubmed/1253326 8- accessed in 29th july 2014

[7]. Cella D, Tross S, Orov E, et al. Mood states of patients after the diagnosis of cancer. J Psychosoc Oncol. 1989;7:45-53. Available from:

http://www.ncbi.nlm.nih.gov/pmc/articles/PM C2967778/\#r54- accessed in 29th july 2014

[8]. DeLisa JA, Gans BM, Walsh NE, William L, Frontera WR, Geiringer SR, et al. Physical medicine and rehabilitation. 4th ed.

Philadelphia: Lippincott Williams \& Wilkins; 2005.

[9]. Morello CM, Leckband SG, Stoner CP, Moorhouse DF, Sahagian GA. Randomized double-blind study comparing the efficacy of gabapentin with amitriptyline on diabetic peripheral neuropathy pain. Arch Intern Med. 1999;159(16):1931-1937.

[10]. Visovsky C, Collins M, Abbott L, Aschenbrenner J, Hart C. Putting Evidence Into Practice. Evidence-Based Interventions for Chemotherapy-Induced Peripheral Neuropathy. Clin J Oncol. Nurs. 2007;11(6):901-913.

[11]. Piano V, Verhagen S, Schalkwijk A, Hekster Y, Kress H, Lanteri-Minet M, et al. Treatment for Neuropathic Pain in Patients with Cancer: Comparative Analysis of Recommendations in National Clinical Practice Guidelines from European Countries. Pain Practice 2014; 14: 1-7. doi: 10.1111/papr.12036.

[12]. Larsson SC, Orsini N, Wolk A. Vitamin B6 and risk of colorectal cancer: a metaanalysis of prospective studies. JAMA. 2010;303(11):1077-1083.

[13]. Hartman TJ, Woodson K, StolzenbergSolomon R, Virtamo J, Selhub J, Barrett MJ, et al. Association of the B-vitamins pyridoxal 5'phosphate $(\mathrm{B}(6)), \mathrm{B}(12)$, and folate with lung cancer risk in older men. Am J Epidemiol. 2001;153(7):688-694.

[14]. Dumitraşcu M, Munteanu C, Lazarescu H. Hidrotherapy. Balneo-Research Journal. 2012;3(1):23-27. Available from: http://bioclima.ro/J315.pdf. - accessed in 29th july 2014

[15]. Justin F Black. Stephen Kishner. Cancer and Rehabilitation. Mar 19, 2013. Available from:

http://emedicine.medscape.com/article/320261 -overview- accessed in 29th july 2014

[16]. American Physical Therapy Association. Lymphedema. How Physical Therapy can help? Available from: http://www.oncologypt.org/pdfs/factsheets/LymphedemaFactSheetFinal.pdfaccessed in 29th july 2014 